\title{
Modeling and OLAP Cubes for Database of Ground and Municipal Water Supply
}

\author{
Taskeen Zaidi ${ }^{1}$, Annapurna Singh ${ }^{2}$, Vipin Saxena $^{1^{*}}$ \\ ${ }^{1}$ Department of Computer Science, B. B. Ambedkar University, Lucknow, India \\ ${ }^{2}$ Department of Environmental Science, B. B. Ambedkar University, Lucknow, India \\ Email: *vsax1@rediffmail.com
}

Received March 13, 2013; revised April 13, 2013; accepted April 20, 2013

Copyright (C) 2013 Taskeen Zaidi et al. This is an open access article distributed under the Creative Commons Attribution License, which permits unrestricted use, distribution, and reproduction in any medium, provided the original work is properly cited.

\begin{abstract}
Modeling plays an important role for the solution of the complex research problems. When the database became large and complex then it is necessary to create a unified model for getting the desired information in the minimum time and to implement the model in a better way. The present paper deals with the modeling for searching of the desired information from a large database by storing the data inside the three dimensional data cubes. A sample case study is considered as a real data related to the ground water and municipal water supply, which contains the data from the various localities of a city. For the demonstration purpose, a sample size is taken as nine but when it becomes very large for number of localities of different cities then it is necessary to store the data inside data cubes. A well known object-oriented Unified Modeling Language (UML) is used to create Unified class and state models. For verification purpose, sample queries are also performed and corresponding results are depicted.
\end{abstract}

Keywords: Modeling; Database; Object-Oriented; Unified Modeling Language; OLAP Data Cubes; Water Supply

\section{Introduction}

In the current scenario, modeling becomes an integral part of the solution of any kind of research problems whether it is related to life sciences, medical sciences, engineering sciences, etc. An object-oriented modeling language is most popular language because of the evolution of the Graphical User Interface (GUI) applications in the computer science filed. On the basis of object-oriented technology, Object Management Group (OMG) [1] has launched the various version of one of the most powerful platform independent modeling language i.e. Unified Modeling Language (UML). It contains various kinds of symbols for drafting a design on a piece of paper. In this connection, Booch and Rambaugh [2] have designed various diagrams for Unified Modeling Language. By the use of UML, the present work is based on the On Line Analytical Processing (OLAP) by designing the OLAP data cubes. The research on the design of three dimensional the data cubes is available in [3]. By the use of the various tools, one can design the OLAP data cubes. In the data cubes information in the form of data is stored along the three axes i.e. $\mathrm{x}, \mathrm{y}$ and $\mathrm{z}$ axis and it supports the object-oriented and structured technologies.

*Corresponding author.
In this work, the data were taken from the observations related to the ground water and municipal water supply of different localities of a city. Let us describe some of the important references related to the status of supply of water. In the current scenario, surface water has polluted due to the various reasons overall known as the weather pollution. The different reasons and challenges in the water technology are described by Barua [4]. Drinking water standards are available in BIS [5], but in the different localities of different cities, the drinking water is going to be polluted daily. This is because of unbalanced chloride and nitrate concentration. The sample database was analyzed on the basis of physiochemical parameters based on standard methods for analysis of water described by Clesceri et al. [6]. World Health Organization [7] has released the various guidelines for the drinking water.

The present work deals with the storage of database related to ground and municipal water supply of drinking water. A concept of UML modeling is used for extracting the information called as Knowledge Discovery in Database (KDD) and it is useful when size of data becomes complex. A three dimensional representation of database in the form of data cubes is designed for storing the large 
database of ground water and municipal water supply. For demonstration purpose, a sample size of nine is considered for storing this database in data cubes and sample queries were performed for the verification purpose.

\section{Background}

\subsection{Unified Modeling Language}

UML is one of the powerful modeling languages and it is a platform independent. One can develop the code very easily by using the object-oriented programming language. It has two views of the problem called as the static and dynamic views. Both contains different types of the diagrams namely class, object diagrams represent the static view of the problem while activity, sequence and state diagrams show the dynamic view of the problem. This modeling language has been developed by Object Management Group.

\subsection{Online Analytical Processing (OLAP) Cube}

It is a multidimensional database which is used by software professionals for optimization of dataware houses. From the dataware houses, data cubes are designed and from the literature it is observed that three dimensional axes are used to design the data cubes and each cell represents the data which may be in the form of text, string or numerals forms. Multidimensional Expression (MDX) Language is used for representing the multidimensional database. The idea of OLAP cube is that it can work faster on the Local Area Network (LAN) or the distributed Wide Area Network (WAN) on which heterogeneous collection of devices can work together. One can get the desired information within fraction of seconds. Really this is a great achievement in the field of the large database and anyone can perform any query and can get result quickly.

\subsection{A Sample Database}

Database is a collection of the information and a real database is taken for ground and municipal water supply of the different localities of a city. For demonstration purpose, a sample size of nine is considered for the database related to ground and municipal water supply and observations [8] are based on the physical chemical characteristics of ground and municipal water quality of various localities of a city. The data is based upon the vicinity of the temperature $20^{\circ} \mathrm{C}$.

$\mathrm{PH}$ was estimated using a potentiometer and it was calibrated using a buffer solution of PH 9.2, PH4 and PH7. Later about $100 \mathrm{ml}$ of sample was taken in a $250 \mathrm{ml}$ beaker and the electrode was dipped to get the $\mathrm{PH}$ value of the sample. For computation of total hardness as per following formula, about $25 \mathrm{ml}$ sample was taken in a
$100 \mathrm{ml}$ flask and a pinch of Erichrome black-T was added to get a vine red colour. The sample was titrated with $0.01 \mathrm{M}$ ethylene diamine tatra aceti Acid to a blue colour.

$$
\text { Total hardness }(\mathrm{mg} / \mathrm{l})=\mathrm{T} \times 1000 \mathrm{xD} / \mathrm{V} \text {; }
$$

where;

$\mathrm{T}=\mathrm{ml}$ of EDTA used.

$\mathrm{D}=\mathrm{mg}$ of $\mathrm{CaCo}_{3}$ equivalent to $1 \mathrm{ml}$ EDTA titrant $(1$ $\mathrm{mg}$ for $0.01 \mathrm{~m}$ EDTA used hear) therefore $\mathrm{D}=1$.

$\mathrm{V}=$ Volume of water sample.

For computation of alkalinity, $50 \mathrm{ml}$ of sample was taken and 2 drops of phenolphthalein indicator was added. An absence of colour showed the presence total alkalinity but absence of phenolphthalein alkalinity. The sample was further titrated with $0.025 \% \mathrm{H}_{2} \mathrm{SO}_{4}$ using mixed indicator. The colour of the solution became pinkat at end point.

$$
\begin{aligned}
& \text { Total alkalinity as }\left(\mathrm{mg} / \mathrm{lCaCO}_{3}\right) \\
& =\mathrm{T} \times \mathrm{N} \times 50 \times 1000 / \text { volume; }
\end{aligned}
$$

where;

$\mathrm{T}=$ Volume of titrant used in $\mathrm{ml}$.

$\mathrm{N}=$ Normality of $\mathrm{H}_{2} \mathrm{SO}_{4}$.

For computation of chloride, $10 \mathrm{ml}$ of sample was taken in a $100 \mathrm{ml}$ flask and 3 drops of $\mathrm{k}_{2} \mathrm{CrO}_{4}$ solution was added to give a yellow colour. The sample was them titrated with $(0.025 \mathrm{~N}) \mathrm{AgNO}_{3}$ to get a brick red colour at end point.

$$
\text { Chloride } \mathrm{mg} / \mathrm{l}=\mathrm{T} \times \mathrm{N} \times 35.45 \times 1000 / \mathrm{V}
$$

where;

$\mathrm{T}=$ Volume of titrant used.

$\mathrm{N}=$ Normality of titrant $\left(\mathrm{AgNO}_{3}\right)$.

$\mathrm{V}=$ Volume of Sample in $\mathrm{ml}$.

For estimation of Nitrate, $0.2 \mathrm{ml}$ of clear sample was

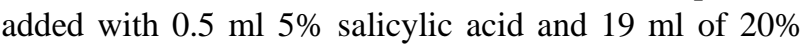
$\mathrm{NaOH}$. A greenish yellow colour indicates the presence of nitrate which is estimated using a spectrophotometer at $410 \mathrm{~nm}$ wavelength.

On all these aspects the practical results are computed and given in Tables 1 and 2, respectively for the ground and municipal water supply.

\section{UML Modeling for KDD}

\subsection{UML Class Diagram}

In the object-oriented technology, UML class diagram shows the static behavior of the system. It can be drawn on a piece of paper and errors can be uncovered during the early stage of software development. Generally, software designer designs such type of diagram for implementation in the object-oriented programming style. A class is defined as group of attributes i.e. variables and 
Table 1. Database of ground water supply (table name: tblgws.Idf).

\begin{tabular}{|c|c|c|c|c|c|c|}
\hline Temperature (Kelvin) & $\mathrm{PH}$ & Total hardness (mg/lit) & Total suspended solids & Chloride (mg/lit) & Total alkalinity (mg/lit) & Nitrate (mg/lit) \\
\hline 293.15 & 7.77 & 180 & 0.035 & 42.5 & 190 & 33.4 \\
\hline 294.15 & 7.76 & 180 & 0.032 & 28.4 & 200 & 16.4 \\
\hline 294.65 & 7.7 & 200 & 0.035 & 85.1 & 214 & 18.1 \\
\hline 294.95 & 7 & 180 & 0.03 & 28.4 & 192 & 53.2 \\
\hline 295.15 & 7.05 & 220 & 0.035 & 70.9 & 234 & 37.9 \\
\hline 295.15 & 7.3 & 140 & 0.28 & 121 & 198 & 26.6 \\
\hline 294.15 & 7.6 & 180 & 0.36 & 21.3 & 228 & 12.7 \\
\hline 294.15 & 7.5 & 160 & 0.34 & 35.5 & 180 & 31.1 \\
\hline 294.44 & 7.46 & 180 & 0.1434 & 54.2 & 205 & 28.7 \\
\hline
\end{tabular}

Table 2. Database of municipal water supply (table name: tblmws.ldf).

\begin{tabular}{|c|c|c|c|c|c|c|}
\hline Temperature (Kelvin) & $\mathrm{PH}$ & Total hardness (mg/lit) & Total suspended solids & Chloride (mg/lit) & Total alkalinity (mg/lit) & Nitrate (mg/lit) \\
\hline 294.15 & 8.25 & 60 & 0.02 & 14.18 & 110 & 20.36 \\
\hline 295.15 & 8.1 & 216 & 0.043 & 14.18 & 196 & 25.17 \\
\hline 295.55 & 7.98 & 200 & 0.036 & 21.27 & 192 & 25.17 \\
\hline 295.55 & 7.53 & 200 & 0.032 & 21.27 & 180 & 24.6 \\
\hline 296.55 & 7.1 & 180 & 0.03 & 70.94 & 212 & 23.19 \\
\hline 296.15 & 7.3 & 180 & 0.3 & 121.4 & 210 & 27.9 \\
\hline 295.15 & 7.99 & 200 & 0.39 & 21.27 & 196 & 14.9 \\
\hline 295.15 & 8.09 & 160 & 0.32 & 21.27 & 204 & 17.81 \\
\hline 294.44 & 7.792 & 174.5 & 0.1464 & 38.22 & 184.5 & 22.39 \\
\hline
\end{tabular}

the methods applied on the attributes. The accessing of the attributes and methods may be private, public or protected. AUML class diagram for accessing of the desired information is shown in the Figure 1. There are six classes namely User, Storage, Handheld devices, KDD, Data cubes and Search pattern. KDD class stands for the Knowledge Discovery Database. By the use of User class, user may login on the handheld device which may be laptop, I-pad, mobile, etc. The user desires to search knowledge database from a large database which is controlled by the class KDD. Search pattern class is responsible for the optimized search technique from the designed data cubes. The searching of the database is faster in comparison of the direct access from the database which is controlled by the Storage class as shown in the Figure 1.

\subsection{UML State Diagram}

The dynamic behavior of the system is represent by the state diagram and in the object-oriented technology,
UML state diagram represents the functioning of the clock of handheld device in which the events are happening as per the forward clock of the device. Figure 2 shows the state diagram for the display of the desired information by the use of data cubes. Initially user enters its id and password on the hand-held device. A large database is converted into the Knowledge Discovery Database (KDD) and thereafter data cubes are designed and user can found the display of the desired database on the device.

\section{Design of OLAP Cube}

The physiochemical characteristics of ground water and municipal water supply are stored in a three dimensional cubes. The three axis $\mathrm{x}, \mathrm{y}, \mathrm{z}$ are represented as hardness, chloride and nitrate, respectively. The database is recorded in each cell of the cube represented along $\mathrm{x}, \mathrm{y}$ and $\mathrm{z}$ axes. The cells can be increased for the finite values of hardness, chloride and nitrate. The above database is represented in the following data cubes for the ground 


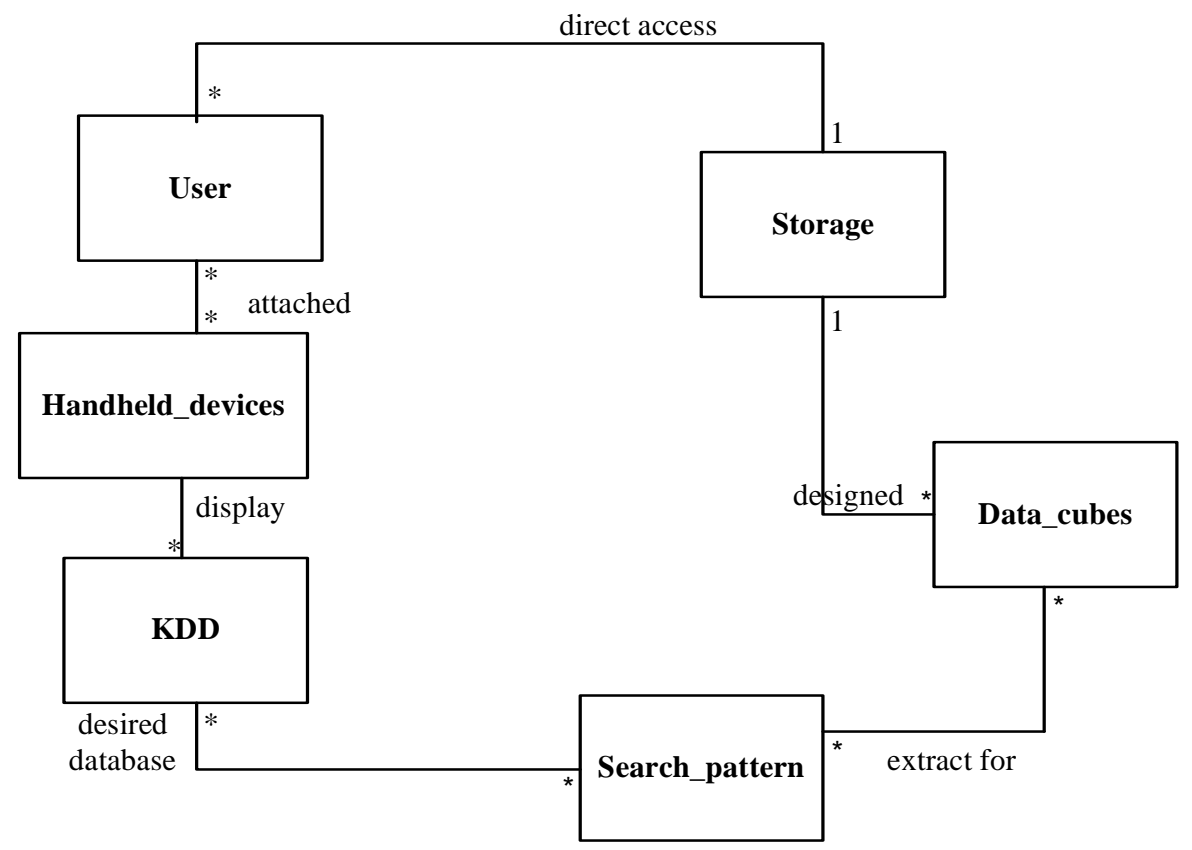

Figure 1. UML class diagram for storage of large database.

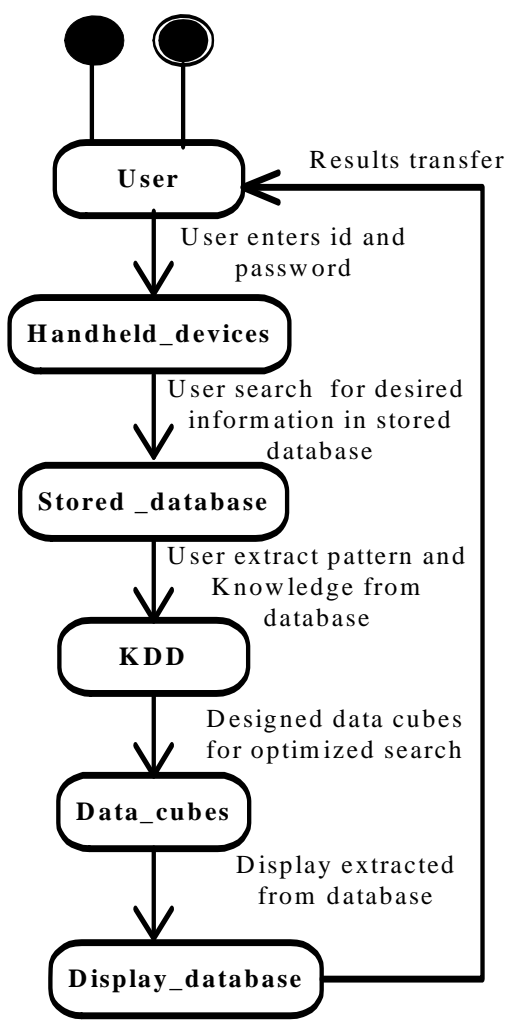

Figure 2. UML state diagram for KDD.

water and municipal water supply in Figures $\mathbf{3}$ and $\mathbf{4}$, respectively. These cubes support both kind of technology i.e. structured and object-oriented technologies. The implementation can be extracted by using object-oriented Unified Modeling Language.
On the above database, sample queries have been performed for the verification of the data from the database and these are described below briefly by the use of SQL Server:

\section{Sample Query-I}

Select Temperature, $\mathrm{PH}$ from tblgws where Totalhardness $=$ "180".

The output of the above query is shown in Table 3.

Sample Query-II

Select Temperature tblmws where Totalhardness = "180" having Chloride = "70.94".

The output of the above query is shown in Table 4.

\section{Conclusion and Future Scope of Work}

From the above work, it is concluded that the modeling of the research problem is necessary for getting the solution of the problem in optimized way. UML is a powerful modeling language as shown above used to design the models for the ground and municipal water supply. If the database is large, then it can be stored inside the data cubes and user can extract the desired database within a fraction of seconds as shown above. Three dimensional storage of database is an excellent way for storing the large and complex database and one can extract the desired data in a few seconds. The data presented in the tables are real data which can be further extended for the number of localities and then transformed in the form of data cubes and one can get the desired information within a few seconds after executing the SQL queries. The other techniques like co-relation, entropy, Gini indexing can be applied for further interpretation of the results. 


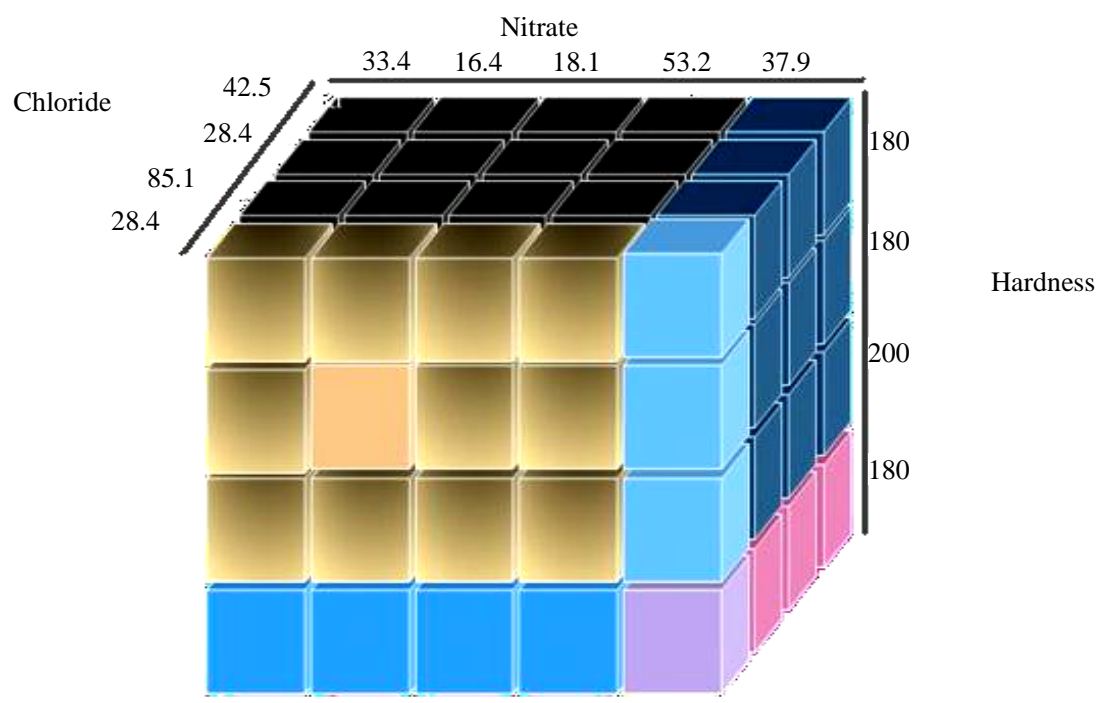

Figure 3. Data cube for ground water supply.

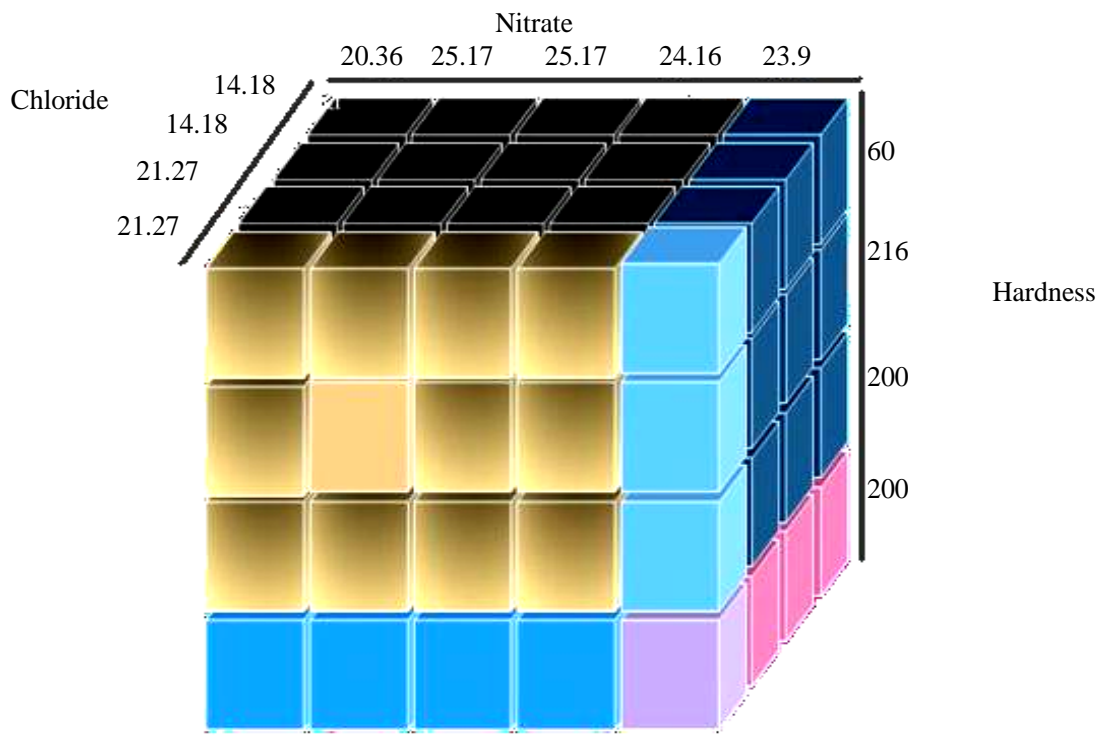

Figure 4. Data cube for municipal water supply.

Table 3. Result of sample query-I.

\begin{tabular}{ccccccc}
\hline Temperature (Kelvin) & PH & Total hardness (mg/lit) & Total suspended solids & Chloride (mg/lit) & Total alkalinity (mg/lit) & Nitrate (mg/lit) \\
\hline 293.15 & 7.77 & 180 & 0.035 & 42.5 & 190 & 33.4 \\
294.15 & 7.76 & 180 & 0.032 & 28.4 & 200 & 16.4 \\
294.95 & 7 & 180 & 0.03 & 28.4 & 192 & 53.2 \\
294.15 & 7.6 & 180 & 0.36 & 21.3 & 228 & 12.7 \\
294.44 & 7.46 & 180 & 0.1434 & 54.2 & 205 & 28.7 \\
\hline
\end{tabular}

Table 4. Result of sample query-II.

\begin{tabular}{ccccccc}
\hline Temperature (Kelvin) & PH & Total hardness (mg/lit) & Total suspended solids & Chloride (mg/lit) & Total alkalinity (mg/lit) & Nitrate (mg/lit) \\
\hline 296.55 & 7.1 & 180 & 0.03 & 70.94 & 212 & 23.19 \\
\hline
\end{tabular}




\section{Acknowledgements}

Thanks are due to University Grants Commission, New Delhi, India for providing financial assistance to carry out the above work.

\section{REFERENCES}

[1] Object Management Group, "Unified Modeling Language Specification,” 2012. http://www.omg.org

[2] G. Booch and J. Rambaugh, "The Unified Modeling Language,” User Guide Addison Wesley, Reading, 1999.

[3] Wikipedia, "OLAP Cube,” 2013. http://en.wikipedia.org/wiki/OLAP_cube

[4] A K. Barua, "Water Technology Management Challenges and Choices in Sustainability of Water Use,” Dominant
Publishers and Distributors, New Delhi, 2001, pp. 2-3.

[5] Bureau of Indian Standards, "Indian Standard Specifications of Drinking Water,” B.S. 2001.

[6] J. A. Camargo and A. Alonso, "Ecological and Toxicological Effects of Inorganic Nitrogen Pollution in Aquatic Ecosystems: A Global Assessment,” Environment International, Vol. 32, No. 6, 2006, pp. 831-849. doi:10.1016/j.envint.2006.05.002

[7] L. S. Clesceri, et al., "Standard Methods for the Examination of Water and Waste Water," American Public Health Association, Washington DC, 1998, p. 19.

[8] A. Singh, et al., "Status of Ground Water and Municipal Water Supply of Lucknow Region U.P.," International Journal of Plant, Animal and Environmental Sciences, Vol. 2, No. 4, 2012, 4 Pages.

\section{Key Points}

- Modeling is necessary to uncover the errors before implementing the solution.

- UML is an object-oriented technique which can be applied in the biological systems also.
- UML shows the static and dynamic behavior of the system.

- OLAP data cubes are used to store the large amount of database as it supports LxMxN data storage for finite values of $\mathrm{L}, \mathrm{M}$ and $\mathrm{N}$. 